\title{
ON NEW SUBCLASS OF HARMONIC UNIVALENT FUNCTIONS ASSOCIATED WITH MODIFIED Q-OPERATOR
}

\section{SHUJAAT ALI SHAH ${ }^{1, *}$, ASGHAR ALI MAITLO ${ }^{1}$, MUHAMMAD AFZAL SOOMRO $^{1}$, KHALIDA INAYAT NOOR ${ }^{2}$}

${ }^{1}$ Department of Mathematics and Statistics, Quaid-i-Awam University of Engineering, Science and Technology, Nawabshah, 67480 Sindh, Pakistan

${ }^{2}$ Department of Mathematics, COMSATS University Islamabad, Islamabad, 45550, Pakistan

${ }^{*}$ Corresponding author: shahglike@yahoo.com

\begin{abstract}
In this article, we introduce new subclasses of harmonic univalent functions associated with the q-difference operator. The modified q-Srivastava-Attiya operator is defined and certain applications of this operator are discussed. We investigate the sufficient condition, distortion result, extreme points and invariance of convex combination of the elements of the subclasses.
\end{abstract}

\section{INTRODUCTION}

A real-valued function $u(x, y)$ is said to be harmonic in a domain $\mathcal{D}$ if it has continuous second order partial derivatives in $\mathcal{D}$ and satisfies

$$
u_{x x}+u_{y y}=0 .
$$

We say that a continuous $f: \Omega(\subset \mathbb{C}) \rightarrow \mathbb{C}$ defined by $f(z)=u(x, y)+i v(x, y)$ is harmonic if both $u(x, y)$ and $v(x, y)$ are real harmonic in $\Omega$. It is observed that every harmonic function $f$ in any simply connected domain $\Omega$ can be written as $f(z)=h(z)+\bar{g}(z)$, where $h$ and $g$ are analytic in $\Omega$, and are called, respectively, the analytic and co-analytic parts of $f$.

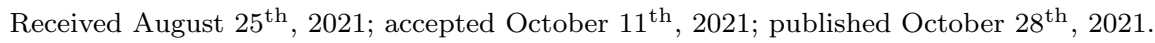

2010 Mathematics Subject Classification. 30C45, 30C55.

Key words and phrases. univalent functions; harmonic function; q-difference operator; the q-Srivastava-Attiya operator.

(C)2021 Authors retain the copyrights of their papers, and all open access articles are distributed under the terms of the Creative Commons Attribution License. 
We denote by $\mathcal{H}$ the class of complex-valued harmonic functions $f=h+\bar{g}$ defined in the open unit disc $\mathcal{U}=\{z:|z|<1\}$ and normalized by $h(0)=g(0)=h^{\prime}(0)-1=0$. Such mappings have the following power series representation

$$
f(z)=z+\sum_{n=2}^{\infty} a_{n} z^{n}+\overline{\sum_{n=2}^{\infty} b_{n} z^{n}}, \quad\left|b_{1}\right|<1 .
$$

It is clear that, when $g(z)$ is identically zero, the class $\mathcal{H}$ reduces to the class $\mathcal{A}$ of normalized analytic functions in $\mathcal{U}$.

Due to Lewy [14], A function $f \in \mathcal{H}$ is locally univalent and sense-preserving in $\mathcal{U}$ if and only if

$$
\left|h^{\prime}(z)\right|>\left|g^{\prime}(z)\right|, \text { for } z \in \mathcal{U}
$$

We denote by $\mathcal{S}_{\mathcal{H}}$ the subclass of $\mathcal{H}$ consisting of all sense-preserving univalent harmonic functions $f$.

Firstly, Clunie et al. [5] was discussed some geometric properties of the class $\mathcal{S}_{\mathcal{H}}$ and its subclasses. Later on, several authors contributed in the study of subclasses of the class $\mathcal{S}_{\mathcal{H}}$, for example, see $[1,3,6,7,9,11,19,20]$.

The theory of $q$-calculus operators are used in describing and solving various problems in applied science such as ordinary fractional calculus, optimal control, $q$-difference and $q$-integral equations, as well as geometric function theory of complex analysis. The fractional $q$-calculus is the $q$-extension of the ordinary fractional calculus and dates back to early $20^{\text {th }}$ century (e.g. see [10] or [2]). For $0<q<1$, the $q$-difference operator was introduced by Jackson [10] and is defined by

$$
\partial_{q} h(z)=\frac{h(z)-h(q z)}{(1-q) z} ; \quad q \neq 1, z \neq 0,
$$

for $q \in(0,1)$ and $h \in \mathcal{A}$ with $h(z)=z+\sum_{n=2}^{\infty} a_{n} z^{n}$.

It is clear that $\lim _{q \rightarrow 1^{-}} \partial_{q} h(z)=h^{\prime}(z)$, where $h^{\prime}(z)$ is the ordinary derivative of the function. It can easily be seen that for $n \in \mathbb{N}=\{1,2,3, .$.$\} and z \in \mathcal{U}$

$$
\partial_{q}\left\{\sum_{n=1}^{\infty} a_{n} z^{n}\right\}=\sum_{n=1}^{\infty}[n]_{q} z^{n-1},
$$

where

$$
[n]_{q}=\frac{1-q^{n}}{1-q}=1+q+q^{2}+\cdots
$$

Recently, in [18], Shah and Noor introduced the $q$-analogue of Srivastava-Attiya operator $J_{q, b}^{s}: \mathcal{A} \rightarrow \mathcal{A}$ by

$$
J_{q, b}^{s} h(z)=z+\sum_{n=2}^{\infty}\left(\frac{[1+b]_{q}}{[n+b]_{q}}\right)^{s} a_{n} z^{n},
$$

where $h \in \mathcal{A}, s \in \mathbb{C}$ and $b \in \mathbb{C} \backslash \mathbb{Z}_{0}^{-}$. It is noted that for $q \rightarrow 1^{-}$in (1.3), then the integral operator studied by the authors in [21] is deduced. Moreover, for particular choices of $s$ and $b$, the operator $J_{q, b}^{s}$ reduces to the $q$-Alexander, $q$-Libera and $q$-Bernardi operators defined in [17]. 
Jahangiri [12] was the first who introduced the $q$-analogue of complex harmonic functions and studied various geometric properties. Nowadays, certain subclasses of $\mathcal{S}_{\mathcal{H}}$ associated with operators and $q$-operators were discussed by the prominent researchers, like $[8,12,13,15,16,22]$. In motivation of above said literature, first we modify the $q$-Srivastava-Attiya operator and then we define some new subclasses of $\mathcal{S}_{\mathcal{H}}$.

For $f=h+\bar{g}$ given by (1.1), we define

$$
J_{q, b}^{s} f(z)=J_{q, b}^{s} h(z)+\overline{J_{q, b}^{s} g(z)}
$$

where $J_{q, b}^{s} h(z)$ is given by $(1.3)$ and

$$
J_{q, b}^{s} g(z)=\sum_{n=1}^{\infty}\left(\frac{[1+b]_{q}}{[n+b]_{q}}\right)^{s} b_{n} z^{n} .
$$

It is observed that, if co-analytic part of $f=h+\bar{g}$ is identically zero, then the modified $q$-Srivastava-Attiya operator defined by (1.4) turn out to be the $q$-Srivastava-Attiya operator introduced in [18].

For $f=h+\bar{g} \in \mathcal{S}_{\mathcal{H}}$, we define a new class $\mathcal{H} \mathcal{S}_{q}(\gamma, \lambda, \beta)$ as follows:

Definition 1.1. Let $f=h+\bar{g} \in \mathcal{S}_{\mathcal{H}}$ and is given by (1.1). Then $f \in \mathcal{H S}_{q}(\gamma, \lambda, \beta)$ if

$$
\Re\left[1+\frac{1}{\gamma}\left\{(1-\lambda) \frac{f(z)}{z}+\lambda\left(\partial_{q} f(z)\right)-1\right\}\right] \geq \beta,
$$

where $\beta \in[0,1), \gamma \in \mathbb{C} \backslash\{0\}, \lambda \in[0,1]$ and $q \in(0,1)$.

Particularly, If $q \rightarrow 1^{-}$, then the class $\mathcal{H} \mathcal{S}_{q}(\gamma, \lambda, \beta)$ reduces to the class, denoted by $\mathcal{H} \mathcal{S}(\gamma, \lambda, \beta)$, of functions $f \in \mathcal{S}_{\mathcal{H}}$ satisfies

$$
\Re\left[1+\frac{1}{\gamma}\left\{(1-\lambda) \frac{f(z)}{z}+\lambda f^{\prime}(z)-1\right\}\right] \geq \beta,
$$

where $\beta \in[0,1), \gamma \in \mathbb{C} \backslash\{0\}$ and $\lambda \in[0,1]$. Moreover, we denote by $\mathcal{H S}(1, \lambda, \beta)=\mathcal{H} \mathcal{S}(\lambda, \beta)$ and $\mathcal{H S}(1,1, \beta)=\mathcal{H S}(\beta)$ the classes of functions $f=h+\bar{g} \in \mathcal{S}_{\mathcal{H}}$ satisfies

$$
\Re\left[(1-\lambda) \frac{f(z)}{z}+\lambda f^{\prime}(z)\right] \geq \beta
$$

and $\Re\left(f^{\prime}(z)\right) \geq \beta$, respectively.

We further define $\overline{\mathcal{H S}}_{q}(\gamma, \lambda, \beta)=\mathcal{H} \mathcal{S}_{q}(\gamma, \lambda, \beta) \cap \overline{\mathcal{S}_{\mathcal{H}}}$, where $\overline{\mathcal{S}_{\mathcal{H}}}$ denote the subclass of $\mathcal{S}_{\mathcal{H}}$ consisting of functions of the type $f(z)=h(z)+\overline{g(z)}$, where

$$
h(z)=z-\sum_{n=2}^{\infty}\left|a_{n}\right| z^{n} \text { and } g(z)=-\sum_{n=1}^{\infty}\left|b_{n}\right| \overline{z^{n}} .
$$

Now, by using modified q-Srivastava-Attiya operator given by (1.4), we define the following.

Definition 1.2. Let $f=h+\bar{g} \in \mathcal{S}_{\mathcal{H}}$, and is given by (1.1). Then, for $s \in \mathbb{R}, b>1, \beta \in[0,1), \gamma \in \mathbb{C} \backslash\{0\}$, $\lambda \in[0,1]$ and $q \in(0,1) . f \in \mathcal{H S}_{q}^{s, b}(\gamma, \lambda, \beta)$ if

$$
\Re\left[1+\frac{1}{\gamma}\left\{(1-\lambda) \frac{J_{q, b}^{s} f(z)}{z}+\lambda\left(J_{q, b}^{s} \partial_{q} f(z)\right)-1\right\}\right] \geq \beta
$$


where $J_{q, b}^{s} f(z)$ is given by (1.4).

Also, we define $\overline{\mathcal{H}}{ }_{q}^{s, b}(\gamma, \lambda, \beta)=\mathcal{H S}_{q}^{s, b}(\gamma, \lambda, \beta) \cap \overline{\mathcal{S}_{\mathcal{H}}}$, where $\overline{\mathcal{S}_{\mathcal{H}}}$ denote the subclass of $\mathcal{S}_{\mathcal{H}}$ consisting of functions given by (1.5).

It is noted that, for $s=0$ we have $\mathcal{H S}_{q}^{s, b}(\gamma, \lambda, \beta)=\mathcal{H \mathcal { S }}$ ( $\left.\gamma, \lambda, \beta\right)$ and $\overline{\mathcal{H S}}_{q}^{s, b}(\gamma, \lambda, \beta)=\overline{\mathcal{H S}}$ ( $\left.\gamma, \lambda, \beta\right)$.

\section{Main Results}

Theorem 2.1. Let a function $f=h+\bar{g} \in \mathcal{S}_{\mathcal{H}}$ given by (1.1) and satisfies

$$
\sum_{n=1}^{\infty}\left\{\left\{1+\left([n]_{q}-1\right) \lambda\right\}\left[\left|a_{n}\right|+\left|b_{n}\right|\right]\right\}\left(\frac{[1+b]_{q}}{[n+b]_{q}}\right)^{s} \leq 1+(1-\beta) \gamma
$$

where $s \in \mathbb{R}, b>1, \beta \in[0,1), \gamma \in \mathbb{C} \backslash\{0\}, \lambda \in[0,1]$ and $q \in(0,1)$. Then $f \in \mathcal{H S}_{q}^{s, b}(\gamma, \lambda, \beta)$. This result is sharp.

Proof. We wish to show that $f=h+\bar{g} \in \mathcal{S}_{\mathcal{H}}$ satisfies (1.6), whenever the coefficients of $f$ satisfies (2.1).

We use the fact that $\Re(w) \geq \xi$ if and only if $|1-\xi+w| \geq|1+\xi-w|$. So it suffices to show that

$$
\begin{array}{r}
\left|1-\beta+1+\frac{1}{\gamma}\left\{(1-\lambda) \frac{J_{q, b}^{s} f(z)}{z}+\lambda\left(J_{q, b}^{s} \partial_{q} f(z)\right)-1\right\}\right| \\
\geq\left|1+\beta-1-\frac{1}{\gamma}\left\{(1-\lambda) \frac{J_{q, b}^{s} f(z)}{z}+\lambda\left(J_{q, b}^{s} \partial_{q} f(z)\right)-1\right\}\right|,
\end{array}
$$

or equivalently,

$$
\begin{array}{r}
\left|(2-\beta) \gamma+(1-\lambda) \frac{J_{q, b}^{s} f(z)}{z}+\lambda\left(J_{q, b}^{s} \partial_{q} f(z)\right)-1\right| \\
-\left|\beta \gamma-(1-\lambda) \frac{J_{q, b}^{s} f(z)}{z}-\lambda\left(J_{q, b}^{s} \partial_{q} f(z)\right)-z\right| \geq 0 .
\end{array}
$$

Using (1.3) and (1.4), from the left hand side, we get

$$
\begin{aligned}
& (2-\beta) \gamma+(1-\lambda)\left\{1+\sum_{n=2}^{\infty}\left(\frac{[1+b]_{q}}{[n+b]_{q}}\right)^{s} a_{n} z^{n-1}+\sum_{n=1}^{\infty}\left(\frac{[1+b]_{q}}{[n+b]_{q}}\right)^{s} \overline{b_{n} z^{n-1}}\right\} \\
& +\lambda\left\{1+\sum_{n=2}^{\infty}\left(\frac{[1+b]_{q}}{[n+b]_{q}}\right)^{s}[n]_{q} a_{n} z^{n-1}+\sum_{n=1}^{\infty}\left(\frac{[1+b]_{q}}{[n+b]_{q}}\right)^{s}[n]_{q} \overline{b_{n} z^{n-1}}\right\}-1 \\
& -\left|\begin{array}{c}
\beta \gamma+(1-\lambda)\left\{1+\sum_{n=2}^{\infty}\left(\frac{[1+b]_{q}}{[n+b]_{q}}\right)^{s} a_{n} z^{n-1}+\sum_{n=1}^{\infty}\left(\frac{[1+b]_{q}}{[n+b]_{q}}\right)^{s} \overline{b_{n} z^{n-1}}\right\} \\
+\lambda\left\{1+\sum_{n=2}^{\infty}\left(\frac{[1+b]_{q}}{[n+b]_{q}}\right)^{s}[n]_{q} a_{n} z^{n-1}+\sum_{n=1}^{\infty}\left(\frac{[1+b]_{q}}{[n+b]_{q}}\right)^{s}[n]_{q} \overline{b_{n} z^{n-1}}\right\}-1
\end{array}\right|,
\end{aligned}
$$


this implies

$$
\begin{aligned}
& \begin{array}{c}
(2-\beta) \gamma+\sum_{n=2}^{\infty}\left\{(1-\lambda)+\lambda[n]_{q}\right\}\left(\frac{[1+b]_{q}}{[n+b]_{q}}\right)^{s} a_{n} z^{n-1} \\
+\sum_{n=1}^{\infty}\left\{(1-\lambda)+\lambda[n]_{q}\right\}\left(\frac{[1+b]_{q}}{[n+b]_{q}}\right)^{s} \overline{b_{n} z^{n-1}}
\end{array} \\
& -\left|\begin{array}{c}
\beta \gamma+\sum_{n=2}^{\infty}\left\{(1-\lambda)+\lambda[n]_{q}\right\}\left(\frac{[1+b]_{q}}{[n+b]_{q}}\right)^{s} a_{n} z^{n-1} \\
+\sum_{n=1}^{\infty}\left\{(1-\lambda)+\lambda[n]_{q}\right\}\left(\frac{[1+b]_{q}}{[n+b]_{q}}\right)^{s} \overline{b_{n} z^{n-1}}
\end{array}\right| \\
& \geq(2-\beta) \gamma-\sum_{n=2}^{\infty}\left\{(1-\lambda)+\lambda[n]_{q}\right\}\left(\frac{[1+b]_{q}}{[n+b]_{q}}\right)^{s}\left|a_{n}\right||z|^{n-1} \\
& -\sum_{n=1}^{\infty}\left\{(1-\lambda)+\lambda[n]_{q}\right\}\left(\frac{[1+b]_{q}}{[n+b]_{q}}\right)^{s}\left|b_{n}\right||z|^{n-1} \\
& -\beta \gamma-\sum_{n=2}^{\infty}\left\{(1-\lambda)+\lambda[n]_{q}\right\}\left(\frac{[1+b]_{q}}{[n+b]_{q}}\right)^{s}\left|a_{n}\right||z|^{n-1} \\
& -\sum_{n=1}^{\infty}\left\{(1-\lambda)+\lambda[n]_{q}\right\}\left(\frac{[1+b]_{q}}{[n+b]_{q}}\right)^{s}\left|b_{n}\right||z|^{n-1} \\
& =2(1-\beta) \gamma\left\{\begin{array}{c}
1-2 \sum_{n=2}^{\infty}\left\{1+\left([n]_{q}-1\right) \lambda\right\}\left(\frac{[1+b]_{q}}{[n+b]_{q}}\right)^{s}\left|a_{n}\right||z|^{n-1} \\
-2 \sum_{n=1}^{\infty}\left\{1+\left([n]_{q}-1\right) \lambda\right\}\left(\frac{[1+b]_{q}}{[n+b]_{q}}\right)^{s}\left|b_{n}\right||z|^{n-1}
\end{array}\right\}
\end{aligned}
$$

The above expression is nonnegative by (2.1). Hence $f \in \mathcal{H S}_{q}^{s, b}(\alpha, \beta)$.

The coefficient bound, given by (2.1), is sharp for the harmonic function

$$
\begin{aligned}
f(z)= & z+\sum_{n=2}^{\infty} \frac{(1-\beta) \gamma}{1+\left([n]_{q}-1\right) \lambda}\left(\frac{[n+b]_{q}}{[1+b]_{q}}\right)^{s} x_{n} z^{n} \\
& +\sum_{n=1}^{\infty} \frac{(1-\beta) \gamma}{1+\left([n]_{q}-1\right) \lambda}\left(\frac{[n+b]_{q}}{[1+b]_{q}}\right)^{s} \bar{y}_{n} \bar{z}^{n}
\end{aligned}
$$

with $\sum_{n=2}^{\infty}\left|x_{n}\right|+\sum_{n=1}^{\infty}\left|y_{n}\right|=1$.

For different choices of parameters, we deduce certain new results as following.

If $s=0$ in Theorem 2.1, then we have a following new result.

Corollary 2.1. Let a function $f(z)=h(z)+\overline{g(z)} \in \mathcal{S}_{\mathcal{H}}$ given by (1.1) and satisfies

$$
\sum_{n=1}^{\infty}\left\{\left\{1+\left([n]_{q}-1\right) \lambda\right\}\left[\left|a_{n}\right|+\left|b_{n}\right|\right]\right\} \leq 1+(1-\beta) \gamma,
$$

where $\beta \in[0,1), \gamma \in \mathbb{C} \backslash\{0\}, \lambda \in[0,1]$ and $q \in(0,1)$. Then $f \in \mathcal{H S}_{q}(\gamma, \lambda, \beta)$. This result is sharp.

If $q \rightarrow 1^{-}$, then Corollary 2.1 reduces to a new result as follows: 
Corollary 2.2. Let a function $f(z)=h(z)+\overline{g(z)} \in \mathcal{S}_{\mathcal{H}}$ given by (1.1) and satisfies

$$
\sum_{n=1}^{\infty}\left\{\{1+(n-1) \lambda\}\left[\left|a_{n}\right|+\left|b_{n}\right|\right]\right\} \leq 1+(1-\beta) \gamma,
$$

where $\beta \in[0,1), \gamma \in \mathbb{C} \backslash\{0\}$ and $\lambda \in[0,1]$. Then $f \in \mathcal{H S}(\gamma, \lambda, \beta)$. This result is sharp.

If we take $\gamma=1$ in Corollary 2.2 then we obtain.

Corollary 2.3. Let a function $f(z)=h(z)+\overline{g(z)} \in \mathcal{S}_{\mathcal{H}}$ given by (1.1) and satisfies

$$
\sum_{n=1}^{\infty}\left\{\{1+(n-1) \lambda\}\left[\left|a_{n}\right|+\left|b_{n}\right|\right]\right\} \leq 2-\beta,
$$

where $\beta \in[0,1)$ and $\lambda \in[0,1]$. Then $f \in \mathcal{H S}(\lambda, \beta)$. This result is sharp.

Moreover, when $\lambda=1$ in Corollary 2.3, we get the sufficient condition for $f$ in $\mathcal{H S}(\beta)$.

Now, we state and prove the necessary and sufficient conditions for the harmonic functions $f=h+\bar{g}$ to be in $\overline{\mathcal{H S}}_{q}^{s, b}(\gamma, \lambda, \beta)$ as following.

Theorem 2.2. Let $f=h+\bar{g} \in \mathcal{S}_{\mathcal{H}}$ given by (1.5). Then $f \in \overline{\mathcal{H S}}_{q}^{s, b}(\gamma, \lambda, \beta)$ if and only if

$$
\sum_{n=1}^{\infty}\left\{\left\{1+\left([n]_{q}-1\right) \lambda\right\}\left[\left|a_{n}\right|+\left|b_{n}\right|\right]\right\}\left(\frac{[1+b]_{q}}{[n+b]_{q}}\right)^{s} \leq 1+(1-\beta) \gamma
$$

where $s \in \mathbb{R}, b>1, \beta \in[0,1), \gamma \in \mathbb{C} \backslash\{0\}, \lambda \in[0,1]$ and $q \in(0,1)$.

Proof. The sufficient condition is obvious from the Theorem 2.1, because $\overline{\mathcal{H S}}_{q}^{s, b}(\gamma, \lambda, \beta) \subset \mathcal{H} \mathcal{S}_{q}^{s, b}(\gamma, \lambda, \beta)$. We need to prove the necessary condition only, that is, if $f \in \overline{\mathcal{H S}}_{q}^{s, b}(\gamma, \lambda, \beta)$, then the coefficients of the function $f=h+\bar{g}$ satisfy the inequality 2.2 .

Let $f \in \overline{\mathcal{H S}}_{q}^{s, b}(\gamma, \lambda, \beta)$. Then, by the definition of $\overline{\mathcal{H S T}}_{q}^{s, b}(\gamma, \lambda, \beta)$, we have

$$
\Re\left[1+\frac{1}{\gamma}\left\{(1-\lambda) \frac{J_{q, b}^{s} f(z)}{z}+\lambda\left(J_{q, b}^{s} \partial_{q} f(z)\right)-1\right\}-\beta\right] \geq 0,
$$

where $s \in \mathbb{R}, b>1, \beta \in[0,1), \gamma \in \mathbb{C} \backslash\{0\}, \lambda \in[0,1]$ and $q \in(0,1)$.

Equivalently, we can write (2.3) as

$$
\Re\left\{(1-\beta) \gamma+(1-\lambda) \frac{J_{q, b}^{s} f(z)}{z}+\lambda\left(J_{q, b}^{s} \partial_{q} f(z)\right)-1\right\} \geq 0 .
$$

Substituting $f=h+\bar{g}$ in (2.4) and employing (1.4) along with (1.5), and also some computation yields

$$
\Re\left[\begin{array}{c}
(1-\beta) \gamma+(1-\lambda)\left\{1-\sum_{n=2}^{\infty}\left(\frac{[1+b]_{q}}{[n+b]_{q}}\right)^{s}\left|a_{n}\right| z^{n-1}-\sum_{n=1}^{\infty}\left(\frac{[1+b]_{q}}{[n+b]_{q}}\right)^{s}\left|b_{n}\right| \bar{z}^{n-1}\right\} \\
+\lambda\left\{1-\sum_{n=2}^{\infty}\left(\frac{[1+b]_{q}}{[n+b]_{q}}\right)^{s}[n]_{q}\left|a_{n}\right| z^{n-1}-\sum_{n=1}^{\infty}\left(\frac{[1+b]_{q}}{[n+b]_{q}}\right)^{s}[n]_{q}\left|b_{n}\right| \bar{z}^{n-1}\right\}-1
\end{array}\right] \geq 0 .
$$

This implies

$$
\Re\left[(1-\beta) \gamma-\sum_{n=2}^{\infty}\left\{1+\left([n]_{q}-1\right) \lambda\right\}\left|a_{n}\right| z^{n-1}-\sum_{n=1}^{\infty}\left\{1+\left([n]_{q}-1\right) \lambda\right\}\left|b_{n}\right| \bar{z}^{n-1}\right] \geq 0 .
$$


The above required condition must hold for all values of $z$ in $\mathcal{U}$. Upon choosing the values of $z$ on the positive real axis where $0 \leq z=r<1$, we must have

$$
(1-\beta) \gamma-\sum_{n=2}^{\infty}\left\{1+\left([n]_{q}-1\right) \lambda\right\}\left|a_{n}\right| r^{n-1}-\sum_{n=1}^{\infty}\left\{1+\left([n]_{q}-1\right) \lambda\right\}\left|b_{n}\right| r^{n-1} \geq 0 .
$$

If the inequality (2.2) does not hold, then the numerator in (2.5) is negative for $r$ sufficiently close to 1 . Hence there exists $z_{0}=r_{0}$ in $(0,1)$ for which the quotient in $(2.5)$ is negative. This contradicts the required condition for $f \in \overline{\mathcal{H S T}}_{q}^{s, b}(\gamma, \lambda, \beta)$ and so the proof is complete.

Next, we want to discuss the distortion bounds for the function $f \in \overline{\mathcal{H S}}_{q}^{s, b}(\gamma, \lambda, \beta)$, which yields a covering result for this class.

Theorem 2.3. If $f \in \overline{\mathcal{H S}}_{q}^{s, b}(\gamma, \lambda, \beta)$ and $|z|=r<1$, then

$$
\left(1-\left|b_{1}\right|\right) r-\operatorname{Tr}^{2} \leq|f(z)| \leq\left(1+\left|b_{1}\right|\right) r+T r^{2},
$$

with

$$
T=\left(\frac{[2+b]_{q}}{[1+b]_{q}}\right)^{s}\left[\frac{(1-\beta) \gamma}{\left([2]_{q}-1\right) \lambda+1}-\frac{1}{\left([2]_{q}-1\right) \lambda+1}\left|b_{1}\right|\right]
$$

Proof. Let $f \in \overline{\mathcal{H S}}_{q}^{s, b}(\gamma, \lambda, \beta)$. Taking absolute value of $f$, we get

$$
\begin{aligned}
|f(z)| \leq & \left(1+\left|b_{1}\right|\right) r+\sum_{n=2}^{\infty}\left(\left|a_{n}\right|+\left|b_{n}\right|\right) r^{2} \\
\leq & \left(1+\left|b_{1}\right|\right) r+\frac{(1-\beta) \gamma[2+b]_{q}^{s}}{\left\{\left([2]_{q}-1\right) \lambda+1\right\}[1+b]_{q}^{s}} \\
& \times \sum_{n=2}^{\infty}\left\{\left(\frac{\left\{\left([2]_{q}-1\right) \lambda+1\right\}[1+b]_{q}^{s}}{(1-\beta) \gamma[2+b]_{q}^{s}}\right)\left(\left|a_{n}\right|+\left|b_{n}\right|\right)\right\} r^{2} \\
\leq & \left(1+\left|b_{1}\right|\right) r+\frac{(1-\beta) \gamma[2+b]_{q}^{s}}{\left\{\left([2]_{q}-1\right) \lambda+1\right\}[1+b]_{q}^{s}} \\
& \sum_{n=2}^{\infty}\left\{\left(\frac{\left\{\left([n]_{q}-1\right) \lambda+1\right\}[1+b]_{q}^{s}}{(1-\beta) \gamma[n+b]_{q}^{s}}\right)\left(\left|a_{n}\right|+\left|b_{n}\right|\right)\right\} r^{2} \\
\leq & \left(1+\left|b_{1}\right|\right) r+\frac{(1-\beta) \gamma[2+b]_{q}^{s}}{\left\{\left([2]_{q}-1\right) \lambda+1\right\}[1+b]_{q}^{s}} \\
& \times\left\{1-\frac{\left|b_{1}\right|}{(1-\beta) \gamma}\right\} r^{2}, \quad(b y(2.2)) \\
\leq & \left(1+\left|b_{1}\right|\right) r+T r^{2},
\end{aligned}
$$

where $T$ is given by (2.6). Hence this is the required right hand inequality. Similarly, one can easily prove the required left hand inequality. 
By making use of the left hand inequality of the above theorem and letting $r \rightarrow 1$, we obtain

Corollary 2.4. (Covering result) If $f \in \overline{\mathcal{H S}}_{q}^{s, b}(\gamma, \lambda, \beta)$, then

$$
\left\{w:|w|<(1-L)-(1-M)\left|b_{1}\right|\right\} \subset f(\mathcal{E})
$$

where $L=\frac{(1-\beta) \gamma[2+b]_{q}^{s}}{\left\{\left([2]_{q}-1\right) \lambda+1\right\}[1+b]_{q}^{s}}$ and $M=\frac{[2+b]_{q}^{s}}{\left\{\left([2]_{q}-1\right) \lambda+1\right\}[1+b]_{q}^{s}}$.

In particular, we obtain the covering results for the subclasses of harmonic functions defined in Definition 1.1 and its special cases.

Now, our task is to determine the extreme points of closed convex hulls of $\overline{\mathcal{H S}}_{q}^{s, b}(\gamma, \lambda, \beta)$ denoted by $\operatorname{clco} \overline{\mathcal{H S}}_{q}^{s, b}(\gamma, \lambda, \beta)$.

Theorem 2.4. A function $f \in \overline{\mathcal{H S}}_{q}^{s, b}(\gamma, \lambda, \beta)$ if and only if

$$
f(z)=\sum_{n=1}^{\infty}\left(X_{n} h_{n}(z)+Y_{n} g_{n}(z)\right)
$$

where $h_{1}(z)=z$,

$$
h_{n}(z)=z-\frac{(1-\beta) \gamma}{1+\left([n]_{q}-1\right) \lambda}\left(\frac{[n+b]_{q}}{[1+b]_{q}}\right)^{s} z^{n} ; \quad(n=2,3, \ldots)
$$

and

$$
g_{n}(z)=z-\frac{(1-\beta) \gamma}{1+\left([n]_{q}-1\right) \lambda}\left(\frac{[n+b]_{q}}{[1+b]_{q}}\right)^{s} \bar{z}^{n} ; \quad(n=1,2,3, \ldots)
$$

with $\sum_{n=1}^{\infty}\left(X_{n}+Y_{n}\right)=1$ and $X_{n}, Y_{n} \geq 0$. Particularly, $\left\{h_{n}\right\}$ and $\left\{g_{s_{n}}\right\}$ are the extreme points of $\overline{\mathcal{H S}}_{q}^{s, b}(\gamma, \lambda, \beta)$

Proof. We assume function $f$ as given by (2.7)

$$
\begin{aligned}
f(z) & =\sum_{n=1}^{\infty}\left(X_{n} h_{n}(z)+Y_{n} g_{n}(z)\right) \\
& =\sum_{n=1}^{\infty}\left(X_{n}+Y_{n}\right) z-\sum_{n=2}^{\infty} X_{n} R_{n} z^{n}-\sum_{n=1}^{\infty} Y_{n} R_{n} \bar{z}^{n}
\end{aligned}
$$

where $R_{n}=\frac{(1-\beta) \gamma[n+b]_{q}^{s}}{\left\{\left([n]_{q}-1\right) \lambda+1\right\}[1+b]_{q}^{s}}$.

Equating (2.8) with (1.5), we get

$$
\left|a_{n}\right|=X_{n} R_{n} \text { and }\left|b_{n}\right|=Y_{n} R_{n} .
$$

Now,

$$
\begin{aligned}
& \sum_{n=2}^{\infty} \frac{\left\{\left([n]_{q}-1\right) \lambda+1\right\}[1+b]_{q}^{s}}{(1-\beta) \gamma[n+b]_{q}^{s}}\left|a_{n}\right|+\sum_{n=1}^{\infty} \frac{\left\{\left([n]_{q}-1\right) \lambda+1\right\}[1+b]_{q}^{s}}{(1-\beta) \gamma[n+b]_{q}^{s}}\left|b_{n}\right| \\
= & \sum_{n=1}^{\infty}\left(X_{n}+Y_{n}\right)-X_{1}=1-X_{1} \leq 1,
\end{aligned}
$$


this implies

$$
\sum_{n=1}^{\infty}\left[\left\{\left([n]_{q}-1\right) \lambda+1\right\}\left[\left|a_{n}\right|+\left|b_{n}\right|\right]\right]\left(\frac{[1+b]_{q}}{[n+b]_{q}}\right)^{s} \leq 1+(1-\beta) \gamma
$$

Thus, by Theorem 2.2, $f \in \overline{\mathcal{H S}}_{q}^{s, b}(\gamma, \lambda, \beta)$. Conversely, let $f \in \overline{\mathcal{H S}}_{q}^{s, b}(\gamma, \lambda, \beta)$. We take

$$
X_{n}=\frac{\left\{\left([n]_{q}-1\right) \lambda+1\right\}[1+b]_{q}^{s}}{(1-\beta) \gamma[n+b]_{q}^{s}}\left|a_{n}\right| ; \quad(n=2,3, \ldots)
$$

and

$$
Y_{n}=\frac{\left\{\left([n]_{q}-1\right) \lambda+1\right\}[1+b]_{q}^{s}}{(1-\beta) \gamma[n+b]_{q}^{s}}\left|b_{n}\right| ; \quad(n=1,2, \ldots)
$$

with $\sum_{n=1}^{\infty}\left(X_{n}+Y_{n}\right)=1$.

We follows our required result by substituting the values of $\left|a_{n}\right|$ and $\left|b_{n}\right|$ from the above relations in $(1.5)$.

Finally, we wish to show that the class $\overline{\mathcal{H S}}_{q}^{s, b}(\gamma, \lambda, \beta)$ is closed under the convex combination of its elements.

Theorem 2.5. The class $\overline{\mathcal{H S}}_{q}^{s, b}(\gamma, \lambda, \beta)$ is closed under the convex combination.

Proof. Let $f_{i_{s}} \in \overline{\mathcal{H S}}_{q}^{s, b}(\gamma, \lambda, \beta),(i=1,2, \ldots)$, with

$$
f_{i}=z-\sum_{n=2}^{\infty}\left|a_{i, n}\right| z^{n}-\sum_{n=1}^{\infty}\left|b_{i, n}\right| \bar{z}^{n}
$$

On using Theorem 2.2, we can write

$$
\left[\sum_{n=1}^{\infty}\left\{\left\{1+\left([n]_{q}-1\right) \lambda\right\}\left[\left|a_{n}\right|+\left|b_{n}\right|\right]\right\}\right]\left(\frac{[1+b]_{q}}{[n+b]_{q}}\right)^{s} \leq 1+(1-\beta) \gamma .
$$

Now,

$$
\sum_{i=1}^{\infty} \varkappa_{i} f_{i}=z-\sum_{n=2}^{\infty}\left(\sum_{i=1}^{\infty} \varkappa_{i}\left|a_{i, n}\right|\right) z^{n}-\sum_{n=2}^{\infty}\left(\sum_{i=1}^{\infty} \varkappa_{i}\left|a_{i, n}\right|\right) \bar{z}^{n} .
$$

To prove our result, we use (2.8) and (2.9)

$$
\begin{aligned}
& \sum_{n=2}^{\infty} \frac{\left\{\left([n]_{q}-1\right) \lambda+1\right\}[1+b]_{q}^{s}}{(1-\beta) \gamma[n+b]_{q}^{s}}\left(\sum_{i=1}^{\infty} \varkappa_{i}\left|a_{i, n}\right|\right) \\
& +\sum_{n=1}^{\infty} \frac{\left\{\left([n]_{q}-1\right) \lambda+1\right\}[1+b]_{q}^{s}}{(1-\beta) \gamma[n+b]_{q}^{s}}\left(\sum_{i=1}^{\infty} \varkappa_{i}\left|b_{i, n}\right|\right) \\
\leq & \sum_{i=1}^{\infty} \varkappa_{i}=1 .
\end{aligned}
$$

Therefore $\sum_{i=1}^{\infty} \varkappa_{i} f_{i_{s}} \in \overline{\mathcal{H S}}_{q}^{s, b}(\gamma, \lambda, \beta)$.

Conflicts of Interest: The author(s) declare that there are no conflicts of interest regarding the publication of this paper. 


\section{REFERENCES}

[1] O.P. Ahuja, J.M. Jahangiri, Noshiro-type harmonic univalent functions, Sci. Math. Jpn. 56 (2002), 1-7.

[2] G.E. Andrews, R. Askey, R. Roy, Special functions, encyclopedia of mathematics and its applications, Cambridge University Press, Cambridge, UK, 1999.

[3] Y. Avci, E. Zlotkiewicz, On harmonic univalent mappings, Ann. Univ. Marie Curie-Sklodowska Sect. A 44 (1990), 1-7.

[4] N.E. Cho, T.H. Kim, Multiplier transformation and strongly close-to-convex functions, Bull. Korean Math. Soc. 40 (2003), $399-410$.

[5] J. Clunie, T. Sheil-Small, Harmonic univalent functions. Ann. Acad. Sci. Fenn., Ser. A 1 Math. 9 (1984), 3-25.

[6] M. Darus, K. Al-Shaqsi, On certain subclass of harmonic univalent functions, J. Anal. Appl. 6 (2008), 17-28.

[7] P.L. Duren, A survey of harmonic mappings in the plane, Texas Tech. Univ. Math. Ser. 18 (1992), 1-15.

[8] J. Dziok, Classes of harmonic functions associated with Ruscheweyh derivatives, Rev. R. Acad. Cienc. Exactas Fís. Nat. Ser. A Mat. (RACSAM) 113 (2019), 1315-1329

[9] J. Dziok, M. Darus, J. Sokół, T. Bulboaca, Generalizations of starlike harmonic functions, C. R. Math. Acad. Sci. Paris 354 (2016), 13-18.

[10] F.H. Jackson, On q-functions and a certain difference operator, Earth Environ. Sci. Trans. R. Soc. Edinburgh. 46 (1908), 253-281.

[11] J.M. Jahangiri, Harmonic functions starlike in the unit disc, J. Math. Anal. Appl. 235 (1999), 470-477.

[12] J.M. Jahangiri, Harmonic univalent functions defined by q-calculus operators, Int. J. Math. Anal. Appl. 5 (2018), $39-43$.

[13] J.M. Jahangiri, G. Murugusundaramoorthy, K. Vijaya, Starlikeness of Rucheweyh type harmonic univalent functions, J. Indian Acad. Math. 26 (2004), 191-200.

[14] H. Lewy, On the non-vanishing of the Jacobian in certain one-to-one mappings, Bull. Amer. Math. Soc. 42 (1936), 689-692.

[15] G. Murugusundramoorthy, J.M. Jahangiri, Ruscheweyh-type harmonic functions defined by q-differential operators, Khayyam J. Math. 5 (2019), 79-88.

[16] K.I. Noor, B. Malik, S.Z.H. Bukhari, Harmonic functions defined by a generalized fractional differential operator, J. Adv. Math. Stud. 2 (2009), 41-52.

[17] K.I. Noor, S. Riaz, M.A. Noor, On q-Bernardi integral operator, TWMS J. Pure Appl. Math. 8 (2017), 3-11.

[18] S.A. Shah, K.I. Noor, Study on q-analogue of certain family of linear operators, Turk. J. Math. 43 (2019), $2707-2714$.

[19] T. Sheil-Small, Constants for planar harmonic mappings, J. London Math. Soc. 2 (1990), 237-248.

[20] H. Silverman, Harmonic univalent functions with negative coefficients, J. Math. Anal. Appl. 220 (1998), $283-289$.

[21] H.M. Srivastava, A.A. Attiya, An integral operator associated with the Hurwitz-Lerch Zeta function and differential subordination, Integral Trans. Spec. Funct. 18 (2007), 207-216.

[22] S. Yalçın, A new class of Sălăgean-type harmonic univalent functions, Appl. Math. Lett. 18 (2005), $191-198$. 